\title{
Analysis of the human chorionic gonadotropin protein at the intact level by HILIC-MS and comparison with RPLC-MS
}

Julien Camperi ${ }^{\mathrm{a}}$, Audrey Combès ${ }^{\mathrm{a}}$, Thierry Fournier ${ }^{\mathrm{b}}$, Valerie Pichon $^{\mathrm{a}, \mathrm{c}}$, Nathalie Delaunay $^{\mathrm{a}}$

a'Laboratory of Analytical, Bioanalytical Sciences and Miniaturization, UMR CBI 8231 ESPCI Paris CNRS, PSL University, Paris, France

${ }^{b}$ Laboratory of Physiopathology and PharmacoToxicology of the Human Placenta, UMR-S 1139 Inserm - University Paris Descartes, Sorbonne Paris Cité, Paris, France

${ }^{\mathrm{c} S}$ Sorbonne Université, Paris, France

Corresponding author: Nathalie Delaunay, nathalie.delaunay@espci.fr; +331 40794651. ORCID: 00000003-3008-0286. Corresponding author institution: LSABM, UMR CBI 8231 CNRS - ESPCI Paris, 10 rue Vauquelin, 75005 Paris, France.

Keywords: high resolution mass spectrometry; human Chorionic Gonadotropin; hydrophilic interaction chromatography; intact protein; reversed-phase liquid chromatography; time-of-flight

\section{Declarations}

Funding: This work has received the support of "Institut Pierre-Gilles de Gennes" (laboratoire d'excellence, "Investissements d'avenir" program ANR-10-IDEX-0001-02 PSL and ANR-10-LABX-31). Availability data and material: data will be available on request.

Abbreviations: ACN, acetonitrile; BPC, base peak chromatogram; FA, formic acid; hCG, human Chorionic Gonadotropin; hCG $\alpha$, alpha subunit of the human Chorionic Gonadotropin; hCG $\beta$, beta subunit of the human Chorionic Gonadotropin; HILIC, hydrophilic interaction liquid chromatography; 
r-hCG, recombinant human Chorionic Gonadotropin; TFA: trifluoroacetic acid. TIC, total ion chromatogram; u-hCG, human Chorionic Gonadotropin isolated from urine of pregnant women. XIC, extracted ion chromatogram.

\section{Abstract}

In the present work, the human Chorionic Gonadotropin (hCG) hormone was characterized for the first time by HILIC coupled to high-resolution (HR) quadrupole/time-of-flight (qTOF) mass spectrometry (MS) at the intact level. This heterodimeric protein, consisting of two subunits (hCG $\alpha$ and $\mathrm{h}(\mathrm{G} \beta$ ), possesses 8 potential glycosylation sites leading to a high number of glycoforms and has a molecular weight of about $35 \mathrm{kDa}$. The HILIC conditions optimized in a first paper but using UV detection were applied here with MS for the analysis of two hCG-based drugs, a recombinant hCG and a hCG isolated from the urine of pregnant women. An Amide column $(150 \times 2.1 \mathrm{~mm}, 2.6 \mu \mathrm{m}$, $150 \AA$ A), a mobile phase composed of acetonitrile and water both containing $0.1 \%$ of trifluoroacetic acid and a temperature of $60^{\circ} \mathrm{C}$ were used. The gradient was from 85 to $40 \% \mathrm{ACN}$ in $30 \mathrm{~min}$. The use of TFA that had been shown to be necessary for the separation of glycoforms caused, as expected, an ion suppression effect in MS that was partially overcome by increasing the amount of protein injected $\left(2 \mu \mathrm{L}\right.$ at $1 \mathrm{mg} \mathrm{mL}^{-1}$ ) and reducing the detection $\mathrm{m} / \mathrm{z}$ range (from 1500 to 300 ). These conditions allowed the detection of different glycoforms of hCG $\alpha$. The performance of the HILICHRMS method was compared to that previously obtained in RPLC-HRMS in terms of number of detected glycoforms, selectivity, and sensitivity. The complementarity and orthogonality of the HILIC and RP modes for the analysis of hCG at the intact level were demonstrated. 


\section{Introduction}

The human Chorionic Gonadotropin (hCG) is an hormone essential for the preservation of the pregnancy and the development of the placenta and fetus [1]. It is a heterodimeric glycoprotein, consisting of an $\alpha(\mathrm{hCG} \alpha)$ and a $\beta$ (hCG $\beta$ ) subunit. The $\alpha$-subunit has identical amino acid sequence to the pituitary gonadotropin hormones such as the follicle-stimulating hormone, the thyroidstimulating hormone, and the luteinizing hormone, containing two $\mathrm{N}$-glycosylation sites $[2,3]$, while the $\beta$-subunit represents the specific part of hCG and has $2 \mathrm{~N}$ - and 4 O-glycosylation sites [4]. Nowadays, it is well-known that changes in the glycosylation pattern of a protein affect its biological activity and half-life. Therefore, the detection and identification of the numerous hCG glycoforms is essential to be able to use one or several of them as biomarkers [5].

With 8 potential glycosylation sites, hCG is a highly heterogeneous protein and the characterization of all its glycoforms constitutes an important analytical challenge. The analysis of the protein at the intact level by liquid chromatography (LC) or capillary electrophoresis (CE) hyphenated with mass spectrometry (MS) is a fast and easy approach that allows the determination of the glycosylation profile [6]. Recently, we optimized for the first time a CE method hyphenated with MS for the separation of hCG glycoforms [7]. The MS analyzer was a triple quadrupole that was useful for comparing glycosylation profiles but prevented the glycoform identification, which requires a highresolution (HR) MS. Then, we developed for the first time a LC separation in reversed phase (RP) mode coupled with quadrupole/time-of flight (qTOF) MS for the analysis of hCG at the intact level [8]. This method led to the separation of some $\alpha$-subunit glycoforms, but did not allow the detection of the hCG $\beta$ glycoforms, perhaps due to a higher heterogeneity of hCG $\beta$ and/or a less efficient ionization with its potential $2 \mathrm{~N}$ - and 4 O-glycans. With regard to selectivity in RPLC, a correlation between the retention time of the hCG $\alpha$ glycoforms and the number of their sialic acids present at the terminal position on the $\mathrm{N}$-glycans antennas was observed. Surprisingly, the retention time of hCG $\alpha$ glycoforms increases with the number of their sialic acids whereas this elution order would be more expected with hydrophilic interaction liquid chromatography (HILIC), which is complementary 
and orthogonal to RPLC $[9,10]$. Indeed, the retention mechanism in HILIC is partially based on partitioning of the compounds between a water-enriched layer formed at the surface of the polar stationary phase and the hydro-organic mobile phase when containing a high content of organic solvent. Additional interactions can then occur between the compounds and the stationary phase including hydrogen bonding, ionic, and dipole-dipole interactions $[11,12]$. The highly organic mobile phase is particularly well adapted to MS detection, leading to an improvements in sensitivity for a large variety of compounds [13]. Therefore, the HILIC mode is nowadays largely used for the analysis of small polar compounds, glycopeptides or N- and O-glycans released from glycoproteins [14-16] and could help to separate the glycoforms of a protein according to their glycan moieties.

For the analysis of glycoproteins at intact level in HILIC, only few studies reported its use [17-24]. Its potential was demonstrated for the analysis of intact neo-glycoproteins obtained after a glycosylation procedure from ribonuclease A, and two antigenic proteins, namely TB10.4 and Ag85B [17-19]. These studies allowed the monitoring of the glycosylation process and glycoprotein stability. More recently, Domínguez-Vega et al. [20] developed, for the first time, a HILIC-HRMS method for assessing the glycosylation pattern of one highly glycosylated protein, erythropoietin having $3 \mathrm{~N}$ - and 1 O-glycosylation sites [20]. Although a partial separation was obtained, the qTOF analyzer allowed the identification of 51 glycoforms, in addition to glycoforms with other post-translational modifications such as succinimide, oxidation, and N-terminal methionine-loss products [20].

With regard to hCG, we optimized a HILIC-UV method to separate its glycoforms [25]. Two hCGbased drugs were successfully analyzed using an amide-based column and a mobile phase containing ACN and water with $0.1 \%$ of trifluoroacetic acid (TFA). The resolution obtained made it possible to distinguish the two drugs thanks to their different glycoforms in number and nature. However, the hyphenation with HRMS is mandatory to significantly improve the level of information about the nature of these hCG glycoforms. Therefore, the potential of HILIC-(qTOF) MS for the hCG characterization is here evaluated and compared to the results obtained with RPLC-(qTOF) MS in terms of selectivity, sensitivity, orthogonality, and complementary. 


\section{Materials and methods}

\subsection{Reagents and analytes}

HPLC-grade acetonitrile (ACN) and formic acid (FA) were supplied by Carlo Erba (Val de Reuil, France). TFA was purchased from Sigma-Aldrich (Saint Quentin Fallavier, France). The ultra-pure water was obtained with a Direct-Q3 UV system (Millipore, Molsheim, France). Ovitrelle ${ }^{\circledR}$ (Organon, Oss, The Netherlands) was presented as a solution containing $500 \mathrm{mg} \mathrm{L}^{-1}$ of hCG (recombinant mammalian cell, r-hCG). Pregnyl ${ }^{\circledR}$ (Serono Europe Ltd, London, UK) is a lyophilized powder containing $5,000 \mathrm{IU}$ of hCG isolated from the urine of pregnant women ( $\mathrm{u}$-hCG) ( $1 \mathrm{IU}$ is equivalent to a concentration of $\left.0.092 \mu \mathrm{g} \mathrm{L} \mathrm{L}^{-1}[26,27]\right)$.

The Ovitrelle ${ }^{\circledast}$ solution was reconstituted in $0.5 \mathrm{~mL}$ of water to obtain a stock solution containing $500 \mu \mathrm{gL}^{-1}$ of $\mathrm{r}$-hCG. For Pregnyl ${ }^{\circledR}$, the lyophilized content of each ampoule was dissolved in $1 \mathrm{~mL}$ of water to obtain a concentration of $460 \mu \mathrm{g} \mathrm{mL}^{-1}$. All the solutions were aliquoted and stored at $-20^{\circ} \mathrm{C}$. Before injection into LC-MS, the hCG aliquots were washed and pre-concentrated to $1 \mathrm{mg} \mathrm{mL}^{-1}$ by centrifugation with ultra-centrifugal units (Merck, Darmstadt, Germany) having a molecular weight cut-off of $10 \mathrm{kDa}$.

\subsection{Instrumentations and columns}

\subsubsection{HILIC-(qTOF) MS}

The HILIC-(qTOF) MS experiments were performed on a bio-compatible 1260 Infinity $^{\text {TM }}$ system composed of a quaternary pump, auto-sampler, column oven, and a diode array UV detector (Agilent Technologies, Les Ulis, France) coupled to a MicrOTOF-Q II Mass Spectrometer (Bruker Daltonics, Wissembourg, France) with an electrospray ionization (ESI) source. An Accucore Amide column (150 x $2.1 \mathrm{~mm}, 2.6 \mu \mathrm{m}, 150 \AA ̊$ ) purchased from ThermoFisher (Le Pecq, France) was used. The mobile phase was composed of acetonitrile and water both containing $0.1 \%$ TFA. The mobile phase gradient was from 85 to $40 \% \mathrm{ACN}$ in $30 \mathrm{~min}$, a plateau for $5 \mathrm{~min}$, and a return to the initial composition within 
$1 \mathrm{~min}$ and an equilibrium step for $15 \mathrm{~min}$. The column temperature was maintained at $60^{\circ} \mathrm{C}$. The injection volume was fixed at $2 \mu \mathrm{L}$, the hCG sample was at $1 \mathrm{mg} \mathrm{mL}^{-1}$ in water, and the flow-rate at $0.4 \mathrm{~mL} \mathrm{~min}{ }^{-1}$. The MS experiments were operated in the positive mode and the mass-detection range was set to $\mathrm{m} / \mathrm{z} 2200-2500$. The parameters of the ESI source were as follows: drying gas $\left(\mathrm{N}_{2}\right)$ flow rate, $9.0 \mathrm{~L} \mathrm{~min}{ }^{-1}$, drying gas temperature, $200^{\circ} \mathrm{C}$, nebulizing gas pressure, $30 \mathrm{psi}$, capillary voltage, 4500 V. The system was controlled by the MicroTOF Control Software (Version 3.4, Bruker Daltonics) and the Hystar (Version 3.2, Bruker Daltonics) software was used to interface the HPLC and MS systems. To ensure optimal conditions for the detection, the calibration of the qTOF was performed once a week using cesium clusters $\left(2 \mathrm{~g} \mathrm{~L}^{-1}\right.$ in water-isopropanol $\left.(1: 1, \mathrm{v} / \mathrm{v})\right)$ allowing a mass accuracy of about $10 \mathrm{ppm}$.

\subsubsection{RPLC-(qTOF) MS}

The RPLC-(qTOF) MS method was previously optimized [8]. It was performed on a 1100 LC system (Agilent Technologies) hyphenated to the same (MicroTOF-Q II) MS detector. The RPLC separation was achieved using an Aeris WIDEPORE XB-C18 column (150 x $2.1 \mathrm{~mm}, 200 \AA$ A packed with $3.6 \mu \mathrm{m}$ core-shell silica-based particles (Phenomenex, Le Pecq, France). The mobile phase was composed of $0.1 \% \mathrm{FA}$ in water and $\mathrm{ACN}$. The mobile phase gradient was an increase from 4.5 to $31.5 \%$ of $\mathrm{ACN}$ in $50 \mathrm{~min}$, a plateau for $5 \mathrm{~min}$, and a return to the initial composition within $1 \mathrm{~min}$ and an equilibrium step for $10 \mathrm{~min}$. The column temperature was maintained at $65^{\circ} \mathrm{C}$, the injection volume was $5 \mu \mathrm{L}$, the hCG sample was at $0.1 \mathrm{mg} \mathrm{mL}^{-1}$ in water, and the flow-rate was set at $0.4 \mathrm{~mL} \mathrm{~min}^{-1}$. The MS detection was carried out with the same parameters as those used for the HILIC experiments, except for the mass detection range set to $\mathrm{m} / \mathrm{z}$ 1000-2500. Table S1 lists MS parameters recommended by the manufacturer (Bruker Daltonics) for the analysis of macromolecules (with $\mathrm{m} / \mathrm{z}>1000$ ) that were used. 


\section{Results and discussions}

\subsection{HILIC-UV vs HILIC-HRMS}

The separation of hCG glycoforms in HILIC-UV was previously optimized, the reliability of the method in terms of retention time and peak area was demonstrated, and its application to two hCG-based drugs showed its potential for fingerprinting approach (see Figure S1 in the supplementary data) [25]. The content and nature of the acidic additive (FA and TFA) and the addition of a volatile salt (ammonium formate) in the mobile phase on the retention and the resolution were studied. It was observed that TFA, an ion-pairing agent, was mandatory to obtain a high number of peaks and led to a nice separation. It is worthwhile to notice that only reagents compatible with MS were investigated since, for identification purposes, the hyphenation with HRMS detection is required and this is the objective of this work. The best HILIC separation was obtained with an amide-based stationary phase and a mobile phase composed of water/ACN containing $0.1 \%$ of TFA. However, no peaks were observed in MS using these conditions and a m/z range of 1000-2500, as we did it in RPLC-MS [8]. This may be due to the presence of TFA in the mobile phase, which induced a dramatic decrease in the MS signal, but our previous study showed that TFA was essential to achieve a good HILIC separation of hCG glycoforms. In addition, TFA has a stabilizing effect on protein species in highly organic solutions and is considered as a good solubilizing agent at low concentrations (e.g. $\sim 0.1 \%$ ) [28]. Therefore, a compromise between LC resolution/protein solubilization and MS sensitivity was done.

To overcome this detection problem, two modifications were considered. First, the injected amount of hCG was increased by increasing the injection volume, because the hCG concentration was already high $\left(1 \mathrm{mg} \mathrm{mL}^{-1}\right)$. In our previous study with UV detection [25], the hCG-based drug samples were dissolved in water and an injection volume of $1 \mu \mathrm{L}$ was used. Even if a water-based sample is not favorable in HILIC, as a partial precipitation of hCG was observed with an ACN content higher than $30 \%$, a fully aqueous sample was maintained and the injection volume was increased to $2 \mu \mathrm{L}$. Secondly, the detection mass range was reduced in order to increase the MS signal intensity, even if 
only a single charge state of the glycoforms was observed instead of three generally. Therefore, different small $\mathrm{m} / \mathrm{z}$ ranges of 300 instead of 1500 were tested and it was observed that the $\mathrm{m} / \mathrm{z}$ range between 2200 and 2500 gave the best signal intensities.

With these conditions, the resulting base peak chromatogram (BPC) is presented in Figure $1 \mathrm{~A}$ analyzing the recombinant hCG-based drug sample (r-hCG).
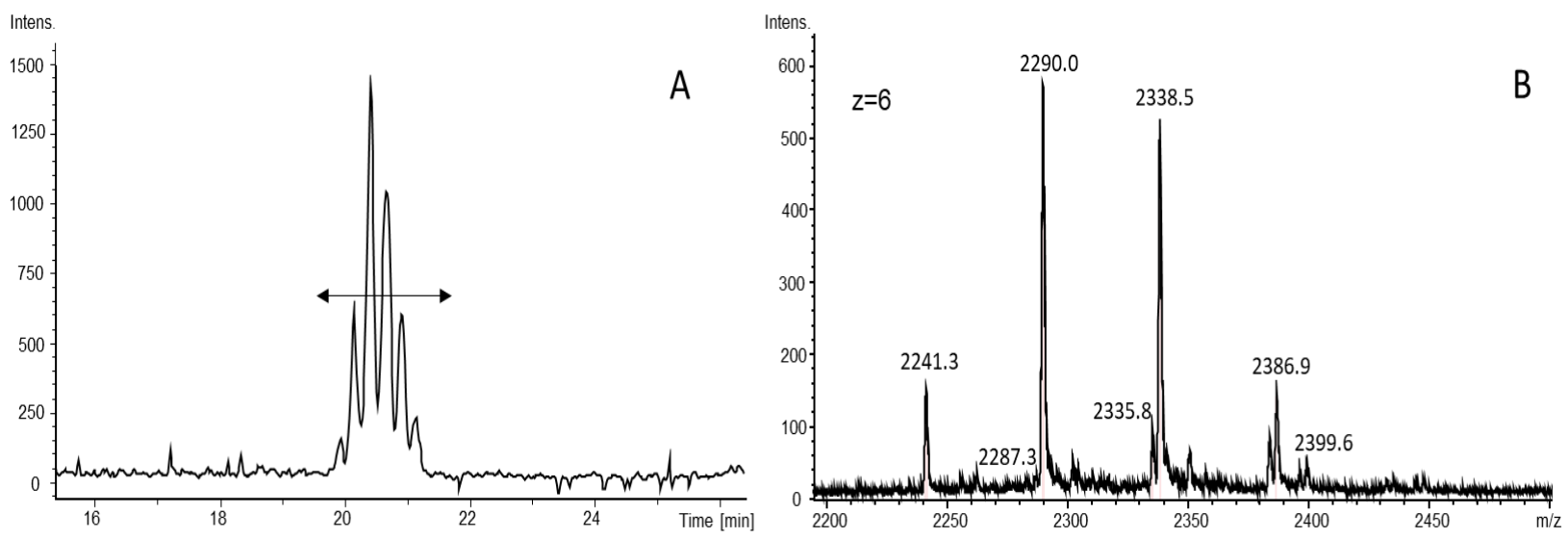

Figure 1: (A) BPC and (B) average MS spectrum between 19.8 and 21.8 min (see arrow) obtained by HILIC-HRMS for r-hCG. Mobile phase: $0.1 \%$ TFA in $\mathrm{H}_{2} \mathrm{O} / \mathrm{ACN}$. Gradient: $85-40 \%$ of ACN with a slope of 1.5\% $\mathrm{min}^{-1}$. Mass range: $2200-2500 \mathrm{~m} / \mathrm{z}$, full scan-positive mode. Temperature: $60^{\circ} \mathrm{C}$. $\mathrm{r}$-hCG samples: $1.0 \mathrm{mg} \mathrm{mL}^{-1}$ prepared in water. Injected volume: $2 \mu \mathrm{L}$. Flow: $0.4 \mathrm{~mL} \mathrm{~min}$.

The presence of 6 peaks between 19.8 and $21.8 \mathrm{~min}$ is observed. It is much less than what was obtained in UV with the same conditions of separation, where at least 10 peaks along 3 elution zones (19.8-21.8, 21.8-23.4, and 23.4-25.2 $\mathrm{min}$ ) were observed [25]. In HILIC-MS, the first zone corresponds to the same first elution zone observed in HILIC-UV, but no peaks were detected in the last two elution zones. Two explanations can be proposed. First, the detection mass range used $(\mathrm{m} / \mathrm{z} 2200-$ 2500) may not be suitable for the detection of glycoforms present in the second and third elution zones. Therefore, different mass ranges were then tested $(\mathrm{m} / \mathrm{z} 100-800, \mathrm{~m} / \mathrm{z} 800-1500$ and, $\mathrm{m} / \mathrm{z}$ 1500-2200), but no peaks were detected. Secondly, these glycoforms are difficult to ionize, which may be due to a high content of glycans and/or the presence of TFA in the mobile phase. Regarding 
the detected peaks in MS, the average spectrum between 19.8 and 21.8 min on the BPC (see arrow Figure $1 \mathrm{~A}$ ) is presented in Figure 1B. As expected, the mass range used ( $\mathrm{m} / \mathrm{z} 2200-2500)$ enabled to detect $\mathrm{m} / \mathrm{z}$ values corresponding to hCG glycoforms with a charge state (z) equal to 6 for all peaks. However, the observation of a single charge state does not allow the application of a conventional deconvolution method for the determination of glycoform mass values requiring 3 charge states.

In conclusion, the applicability of the HILIC-MS method for the analysis of intact hCG is not trivial. Indeed, the presence of TFA, essential to separate the hCG glycoforms as previously demonstrated [25], leads to a very low ionization of glycoforms in MS and thus to a dramatic loss of information on glycoforms present in the last two elution zones which are detected in UV. Nevertheless, the HILICHRMS method gave some $\mathrm{m} / \mathrm{z}$ values corresponding to several $\mathrm{hCG}$ glycoforms in the first elution zone. In order to evaluate the potential of HILIC for hCG characterization, the number of detected glycoforms and the sensitivity of the analysis were then compared to the values obtained by RPLCHRMS [8].

\subsection{Comparison of RPLC and HILIC}

The RPLC-HRMS analysis of $r$-hCG led to about 12 chromatographic peaks (see Figure S2A in the supplementary data) [8]. At least three charge states were observed $(z=9, z=8$, and $z=7)$ with $\mathrm{m} / \mathrm{z}$ values between 1300 and 2200 on the average MS spectrum (Figure S2B) obtained between 28.5 and $32.5 \mathrm{~min}$ on the BPC (see arrow Figure S2A). After deconvolution, these peaks were assigned to glycoforms of the $\alpha$-subunit and no glycoforms of the $\beta$-subunit were detected [8].

The sensitivity between HILIC- and RPLC-HRMS was first compared and it is worthwhile to notice that the qTOF system, r-hCG provider, and LC columns dimensions were the same. In addition, based on the hypothesis that 1 nmole corresponds to $35 \mu \mathrm{g}$ of hCG (MW $\approx 35 \mathrm{kDa}$ ), a quantity of 56.8 pmole was injected in HILIC-HRMS $\left(2 \mu \mathrm{L}\right.$ at $\left.1 \mathrm{mg} \mathrm{mL}^{-1}\right)$, instead of 14.2 pmole in RPLC-HRMS method (5 $\mu \mathrm{L}$ at $100 \mu \mathrm{g} \mathrm{mL}^{-1}$ ). With these conditions, the signal intensity observed on the BPC for the HILIC separation (Figure 1A, approx. 3.0E10 ${ }^{3}$ ) is 10 times less intense than for the RPLC mode (Figure S2A, approx. 
3.0E10 $)$. Therefore, the RPLC-HRMS method appears to be 40 times more sensitive than the HILICHRMS method, despite a larger mass range of detection (m/z 1000-2500 in RPLC-HRMS instead of m/z 2200-2500 in HILIC-HRMS), which clearly demonstrates a dramatic decrease in the sensitivity of the HILIC-HRMS method compared to RPLC-HRMS. As mentioned above, this high sensitivity difference is mainly due to the presence of TFA in HILIC that was required to obtain a high resolution LC separation.

The complementarity of RPLC and HILIC modes was next studied. All the peaks observed on the spectrum obtained in HILIC-HRMS between m/z 2200 and 2500 (Figure 1B) were selected and their eXctracted Ion Chromatograms (XIC) were plotted (Figure 2A).
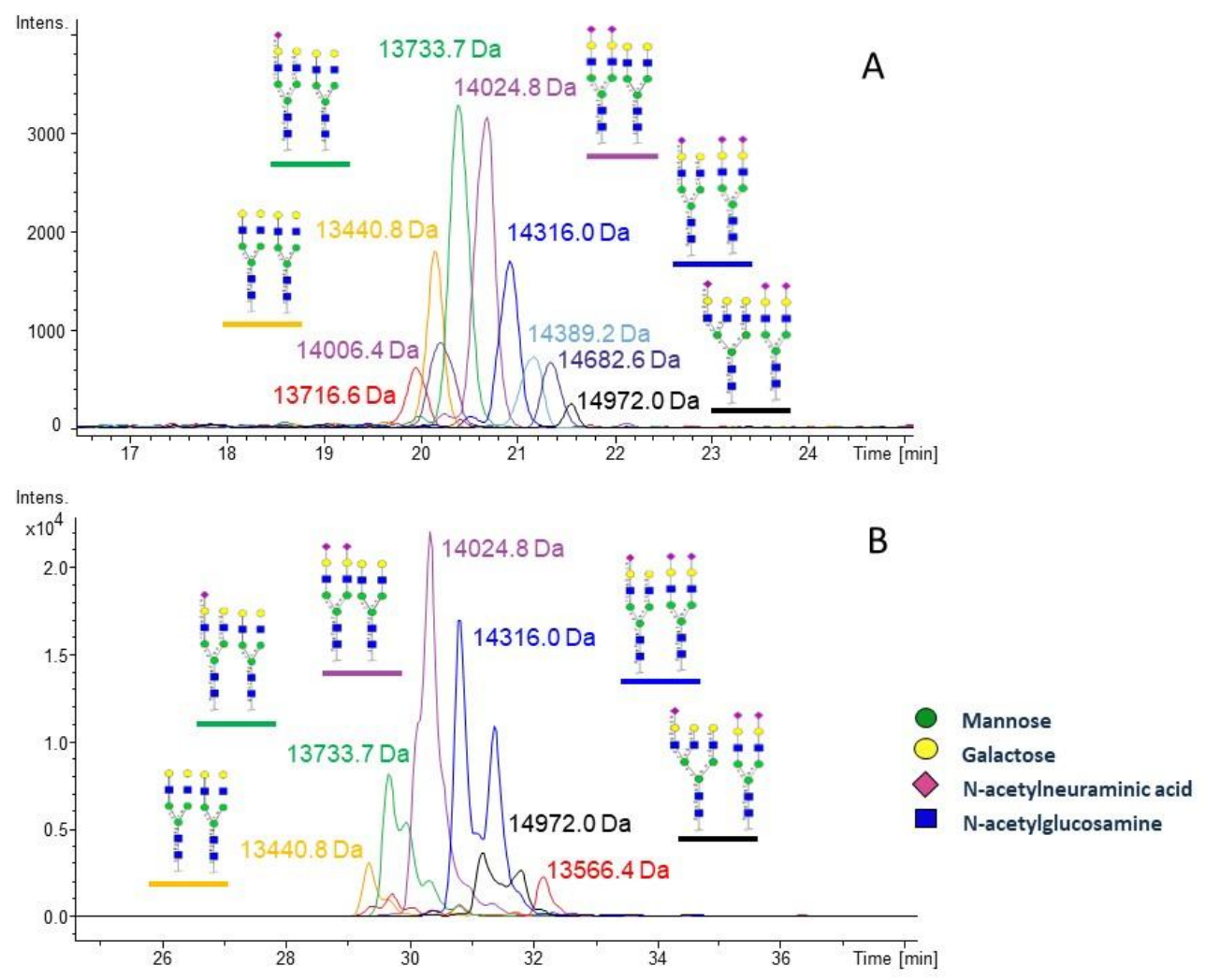

Figure 2: XICs of hCG $\alpha$ glycoforms with N-glycan structures obtained with the analysis of $r$-hCG by (A) HILIC-HRMS or (B) RPLC-HRMS. Conditions: see experimental section. 
The absolute mass values, calculated according to a charge state of $z=6$, are reported. The XIC chromatograms were also plotted for all the peaks observed on the spectrum obtained in RPLC between $\mathrm{m} / \mathrm{z} 1700$ and $1900(\mathrm{z}=8)$ (Figure S2B). They are presented in Figure 2B. First, it is worthwhile to notice the presence of several not resolved peaks associated with a given mass in RPLC (e.g. 14,316.0 Da, Figure 2B), while there is only one peak for a given mass in HILIC (Figures 2A). Although it is difficult to conclude at this stage, two hypotheses will be discussed later.

When comparing Figures $2 \mathrm{~A}$ and $2 \mathrm{~B}$, it is interesting to note that 5 glycoforms with masses of $13,440.8,13,733.7,14,024.8,14,316.0$, and $14,972.0$ Da were detected with both methods. These hCG $\alpha$ glycoforms were previously identified during the RPLC-HRMS study [8] and their glycan structures are represented in Figures $2 \mathrm{~A}$ and 2B. These hCG $\alpha$ glycoforms contain sialylated N-glycans with two antennas, except for one form $(14,972.0 \mathrm{Da})$ having an $\mathrm{N}$-glycan with three antennas. Table 1 shows all the hCGa glycoforms detected during the analysis of r-hCG by HILIC- and RPLC-HRMS. In HILIC, even if one glycoform (13,566.4 Da) was not observed, 4 were detected in addition to RPLC $(13,716.6,14,006.4,14,389.2$, and, $14,682.6 \mathrm{Da}$ ) and 2 out of 4 were identified (a $x$ means that no peak was detected corresponding to its isoform and unidentified that a peak was present on the spectrum but the $\mathrm{N}$-glycans structures were not identified). They contain $2 \mathrm{~N}$-glycans with 2 and 3 antennas. A mass difference corresponding to an $\mathrm{N}$-acetylneuraminic acid was observed between some of them (291 Da between 13,716.6 and 14,006.4 and between 14,389.2 and 14,682.6). It can be noticed that a higher number of glycoforms composed of $\mathrm{N}$-glycans with 3 antennas are detected in HILIC- than in RPLC-HRMS ( 3 in HILIC vs 1 in RPLC). These results demonstrate the complementarity of both separation modes. 
Table 1: List of absolute mass values and N-glycan structures of the $\alpha$-subunit glycoforms of $r$-hCG analyzed by HILIC- or RPLC-HRMS. $x$ : no peaks corresponding to its isoform. Unidentified: a peak is present on the spectrum but the N-glycans structures were not identified. Green circle: mannose; yellow circle: galactose; pink diamond: $\mathrm{N}$-acetylneuraminic acid; blue square: $\mathrm{N}$-acetylglucosamine.

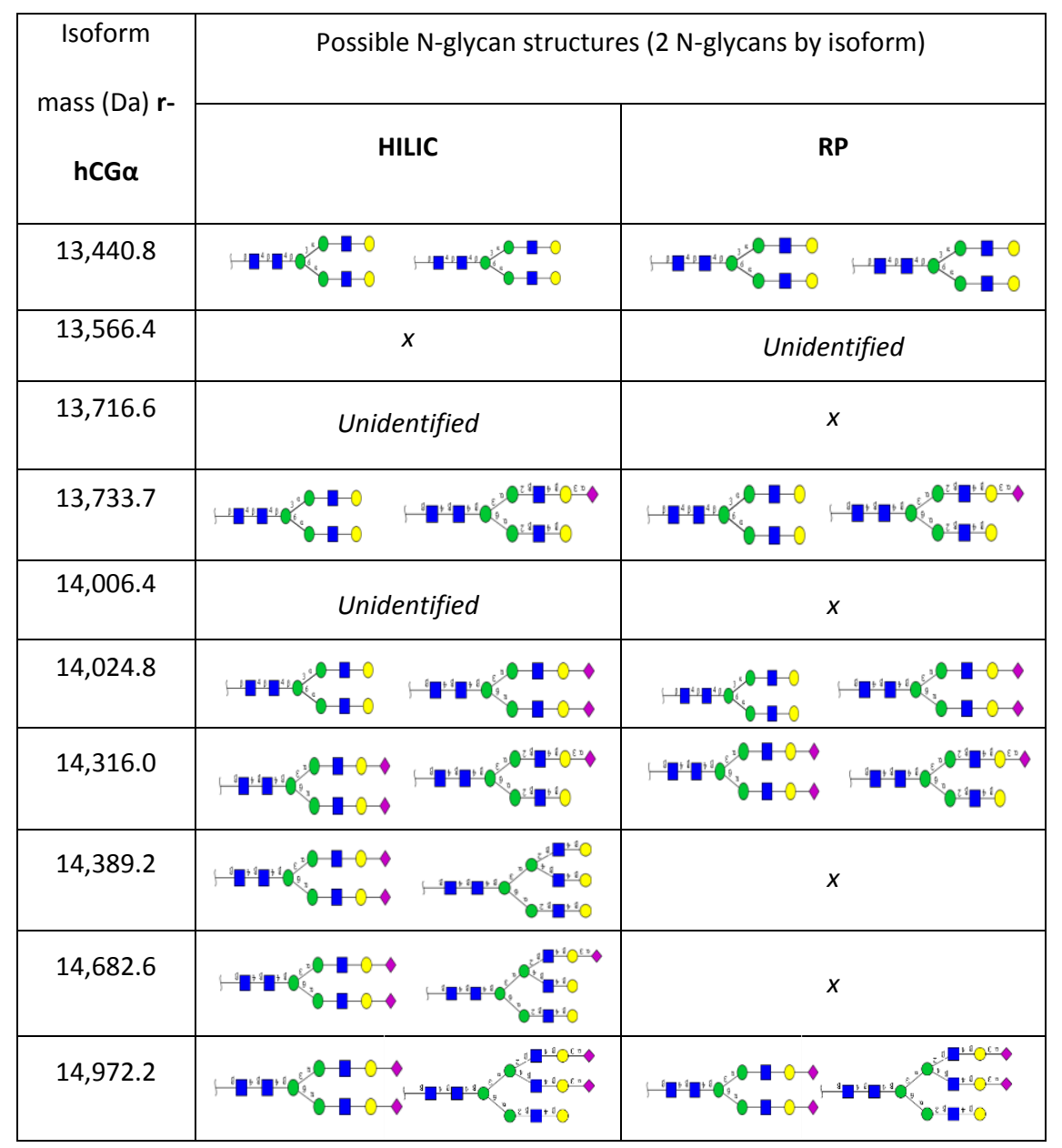

To confirm this complementarity, a second hCG-based drug prepared from urines of pregnant women, u-hCG, was then analyzed by HILIC-HRMS, as it was already carried out by RPLC-HRMS [8]. The resulting BPCS showed in both cases at least 10 peaks with retention times between 19.1 and 20.8 min in HILIC (Figure 3A) and between 28.5 and 32.5 min in RPLC (Figure 3B). As for r-hCG, a decrease in sensitivity was observed in HILIC-HRMS compared to RPLC-HRMS. For all the peaks 
detected on the mass spectrum, the XICs were plotted for each absolute mass value and the glycan structures are presented in Figures $3 \mathrm{C}$ and 3D for HILIC and RPLC, respectively.
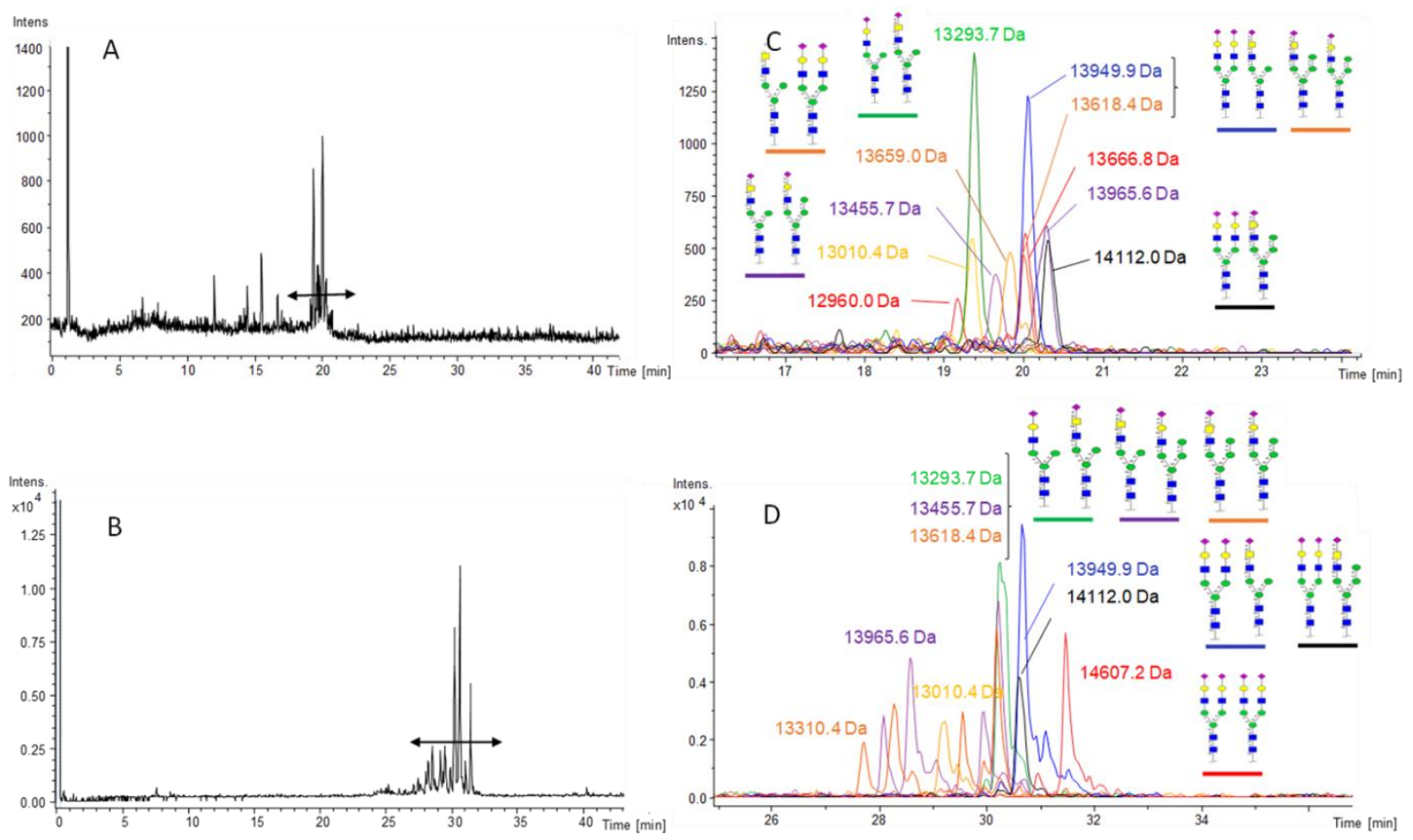

Figure 3: BPCS of u-hCG analyzed by (A) HILIC-HRMS or (B) RPLC-HRMS and XICs of the hCG $\alpha$ glycoforms with their N-glycan structures of u-hCG analyzed by (C) HILIC-HRMS or (D) RPLC-HRMS. Conditions: see experimental section.

Table 2 presents the glycoforms detected with both methods. Seven glycoforms of hCG $\alpha$ were detected in both modes. They are composed of sialylated $\mathrm{N}$-glycans with 1 or/and 2 antennas except for 1 glycoform (13,010.4 Da) which was not identified. Furthermore, 3 additional hCG $\alpha$ glycoforms were detected in HILIC: $12,960.0,13,659.0$, and 13,666.8 Da. Regarding the glycoform with a mass value of $13,659.0 \mathrm{Da}$, the $\mathrm{N}$-glycan structures can be proposed thanks to a mass difference of $291 \mathrm{Da}$ with another already identified glycoform (13,949.9 Da). Unfortunately, the two other hCG $\alpha$ glycoforms were not identified. In RPLC, 2 glycoforms were detected in addition to HILIC: one 
oxidized form (13,310.4 Da) and one with 2 sialylated N-glycans with 2 antennas (Table 2). These results confirm again the complementary of both modes for the identification of hCGa glycoforms.

Table 2: List of absolute mass values and N-glycan structures of the $\alpha$-subunit glycoforms of $\mathrm{u}$-hCG analyzed by HILIC- or RPLC-HRMS. Ox: Oxidized isoforms. $x$ : no peaks corresponding to its isoform. Unidentified: a peak is present on the spectrum but the N-glycans stcutures were not identified. Green circle: mannose; yellow circle: galactose; pink diamond: N-acetylneuraminic acid; blue square: $\mathrm{N}$-acetylglucosamine.

\begin{tabular}{|c|c|c|}
\hline \multirow{2}{*}{$\begin{array}{l}\text { Isoform mass } \\
\text { (Da) u-hCG } \alpha\end{array}$} & \multicolumn{2}{|c|}{ Possible N-glycan structures (2 N-glycans by isoform) } \\
\hline & HILIC & $\mathbf{R P}$ \\
\hline $12,960.0$ & Unidentified & $x$ \\
\hline $13,010.4$ & Unidentified & Unidentified \\
\hline $13,293.7$ & $\ln =0_{0}$ & $\rightarrow=0_{0}$ \\
\hline $13,310.4$ & $x$ & $\mathrm{Ox}+$ \\
\hline $13,455.7$ & 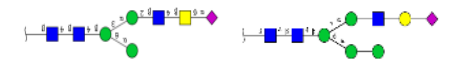 & $=0$ \\
\hline $13,618.4$ & $\ln \log _{0}$ & 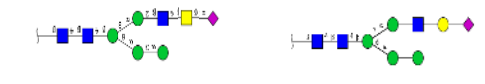 \\
\hline $13,659.0$ & $-0=0$ & $x$ \\
\hline $13,666.8$ & Unidentified & $x$ \\
\hline $13,949.9$ & $\operatorname{loc}_{0}=0$ & $-\log _{0}=0$ \\
\hline $13,965.5$ & $\mathrm{Ox}+\mathrm{a}$ & $\mathrm{Ox}+\mathrm{O}$ \\
\hline $14,112.0$ & $\tan 0$ & $\tan 0$ \\
\hline $14,607.2$ & $x$ & 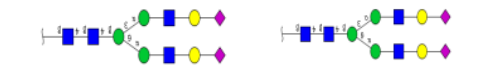 \\
\hline
\end{tabular}


In Tables 1 and 2, it appears that a higher number of hCG $\alpha$ glycoforms was detected in HILIC compared to RPLC for both drugs: 10 and 10 vs 6 and 9 for $r$ - and u-hCG, respectively. As it was already concluded after analyzing both drugs in RPLC [8], despite the additional glycoforms detected in HILIC, none are common to both drugs. As an example, no hCG $\alpha$ glycoforms with 3 antennas were observed for u-hCG but some with a very large number of hybrid glycans. This confirms the strong difference in the glycosylation patterns of the 2 drugs previously mentioned [8].

Finally, the orthogonality of the HILIC and RPLC separation modes was investigated. Figure 4A presents the retention time in HILIC as a function of the retention time in RPLC for the most intense peak of the 4 identified hCGa glycoforms of r-hCG having N-glycans each with 2 antennas. It appears that the number of terminal sialic acids present on this type of glycoform contributes significantly to an increase in its retention time in both modes, which could indicate a poor orthogonality of these methods for the r-hCG characterization. A similar representation was done with the glycoforms identified during the analysis of $\mathrm{u}$-hCG with both methods (Figure 4B). According to Tables 1 and 2, the structures of the corresponding N-glycans are different for u-hCG since at least one out of the four antennas is based on mannose. It appears here that the elution order in HILIC does not seem to be related to the number of sialic acids of the glycoforms. In this case, some glycoforms vary only in the number of mannose sugars they contain, such as $13,293.7,13,455.7$, and $13,618.4$ Da having 6,7 , and 8 mannose sugars, respectively. They co-elute in RPLC but not in HILIC. Looking at glycoforms with similar $\mathrm{N}$-glycan structures but where a mannose has been replaced by an antenna (one $\mathrm{N}$ acetylglucosamine plus one galactose plus one $\mathrm{N}$-acetylneuraminic acid) on one of the two N-glycans, such as the glycoforms of $13,455.7$ and $13,949.9 \mathrm{Da}$, respectively, they co-elute in HILIC but not in RPLC. This clearly demonstrates that HILIC and RPLC are orthogonal separation modes for the separation of u-hCG glycoforms, which have a more heterogeneous composition than $\mathrm{r}$-hCG glycoforms.

As seen previously, it is also important to notice the presence of several peaks associated with a given mass in RPLC for both drugs (see Figure $2 B$ and $3 D$ ), while there is only one peak for a given 
mass in HILIC (Figures 2A and 3C). This difference between both modes clearly appears also in Figures $4 C$ and 4D, where all peaks for one mass were plotted for $r$ - and $u-h C G$, respectively. Two explanations can be proposed. First, the different peaks could correspond to different isomers varying by the localization of the sialic acids on the ends of glycans. However, this hypothesis cannot be retained because several peaks were also observed for non-sialylated glycoforms, having a mass value of $13440.8 \mathrm{Da}$, as shown in Figure $2 \mathrm{~B}$.
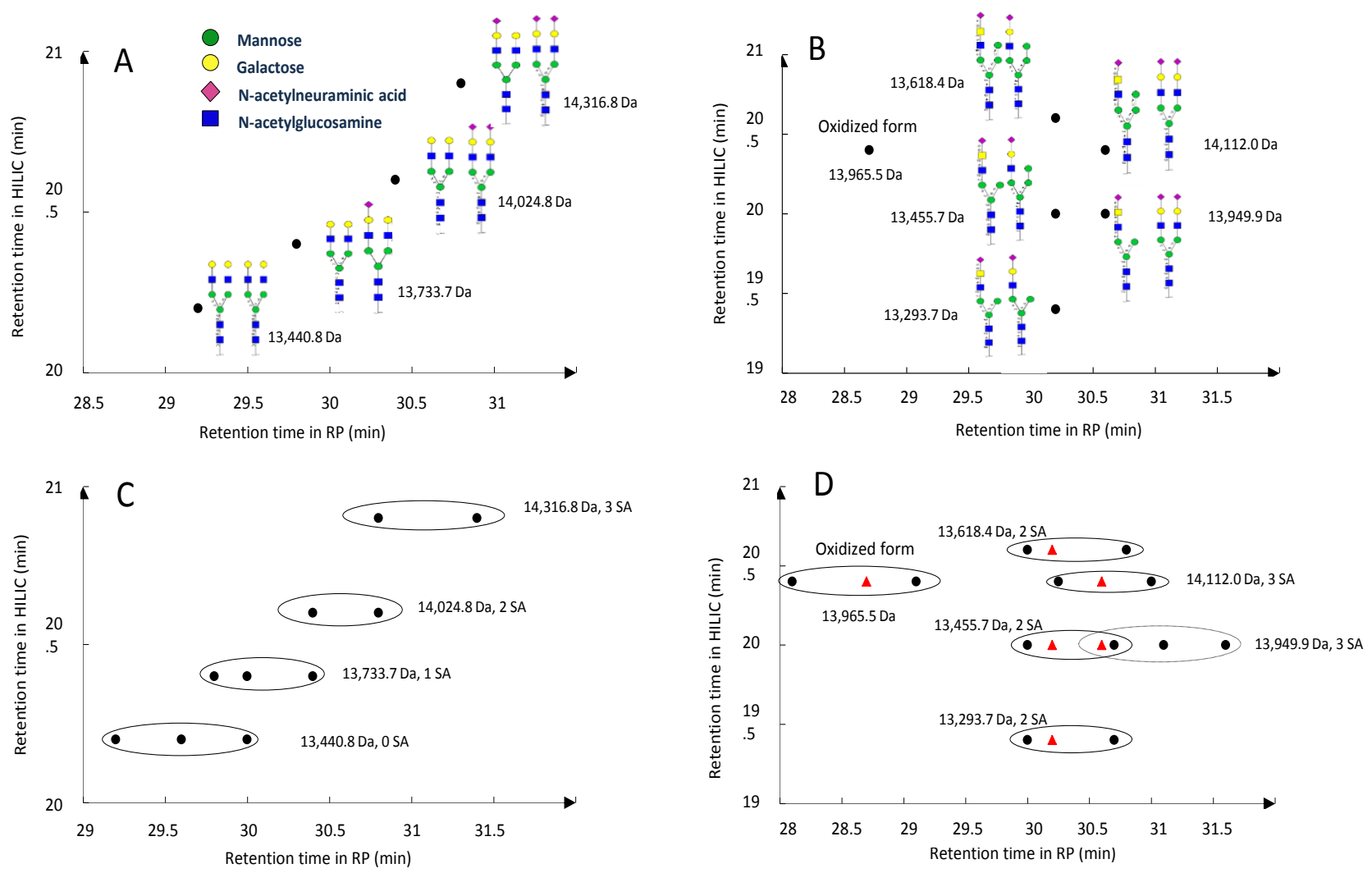

Figure 4: Retention times in HILIC as a function of retention times in RPLC for the common hCG $\alpha$ glycoforms of $r-h C G(A, C)$ and $u-h C G(B, D)$. (A, B) Only the retention time of the most intense peak for each mass value was selected. (C, D) The retention times of all the peaks of a given mass value were selected. The absolute mass of each isoform and its number of sialic acid (SA) are given. In $D, a$ red triangle corresponds to the most intense peak for a given mass value. 
Secondly, the main differences between RPLC and HILIC are the presence of $0.1 \%$ TFA in the HILIC mobile phase instead of formic acid in RPLC and the nature of the stationary phase. Indeed, the injection solvent $\left(\mathrm{H}_{2} \mathrm{O}\right)$, the flow rate $\left(0.4 \mathrm{~mL} \mathrm{~min}^{-1}\right)$, and the $\mathrm{H}_{2} \mathrm{O} / \mathrm{ACN}$-based mobile phase are the same. Even the column temperature was almost identical $\left(65^{\circ} \mathrm{C}\right.$ instead $\left.60^{\circ} \mathrm{C}\right)$. It was already observed that TFA can induce the dissociation of the two subunits of hCG, since they are not covalently linked [4]. Therefore, it could be assumed that the TFA present in the HILIC mobile phase leads to the dissociation of the two subunits during their analysis, giving different retention times for the $\alpha$ - and $\beta$-subunits. This is in agreement with experimental results. Indeed, as observed above, the elution zone observed in HILIC-HRMS corresponds to glycoforms of the $\alpha$-subunit and the two other elution zones observed in our previous experiments in HILIC-UV that are not detected in HILIC-HRMS could correspond to glycoforms of the $\beta$-subunit [8]. Unfortunately, it was impossible to confirm this hypothesis with UV detection and the too low MS signal intensity, due to the presence of TFA and the difficulty to ionize highly glycosylated glycoforms of the $\beta$-subunit that have potentially $2 \mathrm{~N}$ - and 4 O-glycans and should be therefore also more heterogeneous. In RPLC-HRMS, the absence of TFA in the mobile phase and the observation of different chromatographic peaks for one single mass of hCG $\alpha$ could be explained by the fact that the dimeric form of hCG is preserved during the separation. One single hCG $\alpha$ glycoform, which can be more easily ionized with only two N-glycosylation sites, could be associated with different hCG $\beta$ glycoforms, which are not well ionized and consequently not detected in MS [8]. However, it is still not possible to conclude at this stage of the hCG study.

\section{Conclusion and perspectives}

For the first time, a HILIC-HRMS method for the characterization of the highly glycosylated hCG protein at the intact level was developed. Despite the presence of TFA in the mobile phase, which affects the performance of the MS detection while favoring the separation resolution, the HILICHRMS method allowed the identification of different hCGa glycoforms in 2 hCG-based drugs. HILIC allowed the detection of a slightly higher number of hCG $\alpha$ isoforms than RPLC. However, both are 
complementary because some isoforms were only detected in RPLC or HILIC and, even more so, they are orthogonal. Several perspectives can be considered, such as improving sensitivity in HILIC-HRMS or developing two-dimensional approaches coupling HILIC in the first dimension and RPLC in the second. 


\section{References}

[1] L.A. Cole, hCG physiology, Placenta. 34 (2013) 1257. https://doi.org/10.1016/j.placenta.2013.02.011.

[2] M.J. Kessler, T. Mise, R.D. Ghai, O.P. Bahl, Structure and location of the O-glycosidic carbohydrate units of human chorionic gonadotropin, J. Biol. Chem. 254 (1979) 7909-7914.

[3] L. Liu, D. Leaman, M. Villalta, R.M. Roberts, Silencing of the gene for the alpha-subunit of human chorionic gonadotropin by the embryonic transcription factor Oct-3/4, Mol. Endocrinol. Baltim. Md. 11 (1997) 1651-1658. https://doi.org/10.1210/mend.11.11.9971.

[4] C. Liu, L.D. Bowers, Mass spectrometric characterization of the beta-subunit of human chorionic gonadotropin, J. Mass Spectrom. JMS. 32 (1997) 33-42. https://doi.org/10.1002/(SICI)10969888(199701)32:1<33::AID-JMS446>3.0.CO;2-X.

[5] J. Guibourdenche, K. Handschuh, V. Tsatsaris, P. Gerbaud, M.C. Leguy, F. Muller, D. Evain Brion, T. Fournier, Hyperglycosylated hCG Is a Marker of Early Human Trophoblast Invasion, J. Clin. Endocrinol. Metab. 95 (2010) E240-E244. https://doi.org/10.1210/jc.2010-0138.

[6] J. Camperi, V. Pichon, N. Delaunay, Separation Methods hyphenated to Mass Spectrometry for the Characterization of the Protein Glycosylation at the Intact Level, J. Pharm. Biomed. Anal. (2019) 112921. https://doi.org/10.1016/j.jpba.2019.112921.

[7] J. Camperi, B. De Cock, V. Pichon, A. Combes, J. Guibourdenche, T. Fournier, Y. Vander Heyden, D. Mangelings, N. Delaunay, First characterizations by capillary electrophoresis of human Chorionic Gonadotropin at the intact level, Talanta. 193 (2019) 77-86. https://doi.org/10.1016/j.talanta.2018.09.095.

[8] J. Camperi, A. Combes, J. Guibourdenche, D. Guillarme, V. Pichon, T. Fournier, N. Delaunay, An attempt to characterize the human Chorionic Gonadotropin protein by reversed phase liquid chromatography coupled with high-resolution mass spectrometry at the intact level, J. Pharm. Biomed. Anal. (2018). https://doi.org/10.1016/j.jpba.2018.07.056.

[9] A. Periat, S. Fekete, A. Cusumano, J.-L. Veuthey, A. Beck, M. Lauber, D. Guillarme, Potential of hydrophilic interaction chromatography for the analytical characterization of protein biopharmaceuticals, J. Chromatogr. A. 1448 (2016) 81-92. https://doi.org/10.1016/j.chroma.2016.04.056.

[10] T. Tetaz, S. Detzner, A. Friedlein, B. Molitor, J.-L. Mary, Hydrophilic interaction chromatography of intact, soluble proteins, J. Chromatogr. A. 1218 (2011) 5892-5896. https://doi.org/10.1016/j.chroma.2010.09.027.

[11] B. Buszewski, S. Noga, Hydrophilic interaction liquid chromatography (HILIC) - a powerful separation technique, Anal. Bioanal. Chem. 402 (2012) 231-247. https://doi.org/10.1007/s00216-011-5308-5.

[12] M.R. Gama, R.G. da Costa Silva, C.H. Collins, C.B.G. Bottoli, Hydrophilic interaction chromatography, TrAC Trends Anal. Chem. 37 (2012) 48-60. https://doi.org/10.1016/j.trac.2012.03.009.

[13] H.P. Nguyen, K.A. Schug, The advantages of ESI-MS detection in conjunction with HILIC mode separations: Fundamentals and applications, J. Sep. Sci. 31 (2008) 1465-1480. https://doi.org/10.1002/jssc.200700630.

[14] Y. Mechref, D.C. Muddiman, Recent advances in glycomics, glycoproteomics and allied topics, Anal. Bioanal. Chem. 409 (2017) 355-357. https://doi.org/10.1007/s00216-016-0093-9.

[15] J. Bones, S. Mittermayr, N. O’Donoghue, A. Guttman, P.M. Rudd, Ultra Performance Liquid Chromatographic Profiling of Serum N -Glycans for Fast and Efficient Identification of Cancer Associated Alterations in Glycosylation, Anal. Chem. 82 (2010) 10208-10215. https://doi.org/10.1021/ac102860w.

[16] Y. Takegawa, K. Deguchi, H. Ito, T. Keira, H. Nakagawa, S.-I. Nishimura, Simple separation of isomeric sialylated N-glycopeptides by a zwitterionic type of hydrophilic interaction chromatography, J. Sep. Sci. 29 (2006) 2533-2540. https://doi.org/10.1002/jssc.200600133.

[17] F. Rinaldi, S. Tengattini, E. Calleri, T. Bavaro, L. Piubelli, L. Pollegioni, G. Massolini, C. Temporini, Application of a rapid HILIC-UV method for synthesis optimization and stability studies of immunogenic neo -glycoconjugates, J. Pharm. Biomed. Anal. 144 (2017) 252-262. https://doi.org/10.1016/j.jpba.2017.03.052.

[18] S. Tengattini, E. Domínguez-Vega, C. Temporini, T. Bavaro, F. Rinaldi, L. Piubelli, L. Pollegioni, G. Massolini, G.W. Somsen, Hydrophilic interaction liquid chromatography-mass spectrometry as a new tool for the characterization of intact semi-synthetic glycoproteins, Anal. Chim. Acta. 981 (2017) 94-105. https://doi.org/10.1016/j.aca.2017.05.020. 
[19] A. Pedrali, S. Tengattini, G. Marrubini, T. Bavaro, P. Hemström, G. Massolini, M. Terreni, C. Temporini, Characterization of Intact Neo-Glycoproteins by Hydrophilic Interaction Liquid Chromatography, Molecules. 19 (2014) 9070-9088. https://doi.org/10.3390/molecules19079070.

[20] E. Domínguez-Vega, S. Tengattini, C. Peintner, J. van Angeren, C. Temporini, R. Haselberg, G. Massolini, G.W. Somsen, High-resolution glycoform profiling of intact therapeutic proteins by hydrophilic interaction chromatography-mass spectrometry, Talanta. 184 (2018) 375-381. https://doi.org/10.1016/j.talanta.2018.03.015.

[21] Z. Zhang, Z. Wu, M.J. Wirth, Polyacrylamide brush layer for hydrophilic interaction liquid chromatography of intact glycoproteins, J. Chromatogr. A. 1301 (2013) 156-161. https://doi.org/10.1016/j.chroma.2013.05.076.

[22] G. van Schaick, B.W.J. Pirok, R. Haselberg, G.W. Somsen, A.F.G. Gargano, Computer-aided gradient optimization of hydrophilic interaction liquid chromatographic separations of intact proteins and protein glycoforms, J. Chromatogr. A. 1598 (2019) 67-76. https://doi.org/10.1016/j.chroma.2019.03.038.

[23] A.F.G. Gargano, O. Schouten, G. van Schaick, L.S. Roca, J.H. van den Berg-Verleg, R. Haselberg, M. Akeroyd, N. Abello, G.W. Somsen, Profiling of a high mannose-type N-glycosylated lipase using hydrophilic interaction chromatography-mass spectrometry, Anal. Chim. Acta. 1109 (2020) 69-77. https://doi.org/10.1016/j.aca.2020.02.042.

[24] A.F.G. Gargano, L.S. Roca, R.T. Fellers, M. Bocxe, E. Domínguez-Vega, G.W. Somsen, Capillary HILIC-MS: A New Tool for Sensitive Top-Down Proteomics, Anal. Chem. 90 (2018) 6601-6609. https://doi.org/10.1021/acs.analchem.8b00382.

[25] J. Camperi, V. Pichon, T. Fournier, N. Delaunay, First profiling in hydrophilic interaction liquid chromatography of intact human chorionic gonadotropin isoforms, J. Pharm. Biomed. Anal. 174 (2019) 495-499. https://doi.org/10.1016/j.jpba.2019.06.014.

[26] P. Berger, A.J. Lapthorn, The molecular relationship between antigenic domains and epitopes on hCG, Mol. Immunol. 76 (2016) 134-145. https://doi.org/10.1016/j.molimm.2016.06.015.

[27] P. Berger, C. Sturgeon, J.M. Bidart, E. Paus, R. Gerth, M. Niang, A. Bristow, S. Birken, U.H. Stenman, The ISOBM TD-7 Workshop on hCG and related molecules. Towards user-oriented standardization of pregnancy and tumor diagnosis: assignment of epitopes to the three-dimensional structure of diagnostically and commercially relevant monoclonal antibodies directed against human chorionic gonadotropin and derivatives, Tumour Biol. J. Int. Soc. Oncodevelopmental Biol. Med. 23 (2002) 1-38. https://doi.org/48686.

[28] B. Bobály, V. D’Atri, A. Beck, D. Guillarme, S. Fekete, Analysis of recombinant monoclonal antibodies in hydrophilic interaction chromatography: A generic method development approach, J. Pharm. Biomed. Anal. 145 (2017) 24-32. https://doi.org/10.1016/j.jpba.2017.06.016. 


\section{Supplementary data}

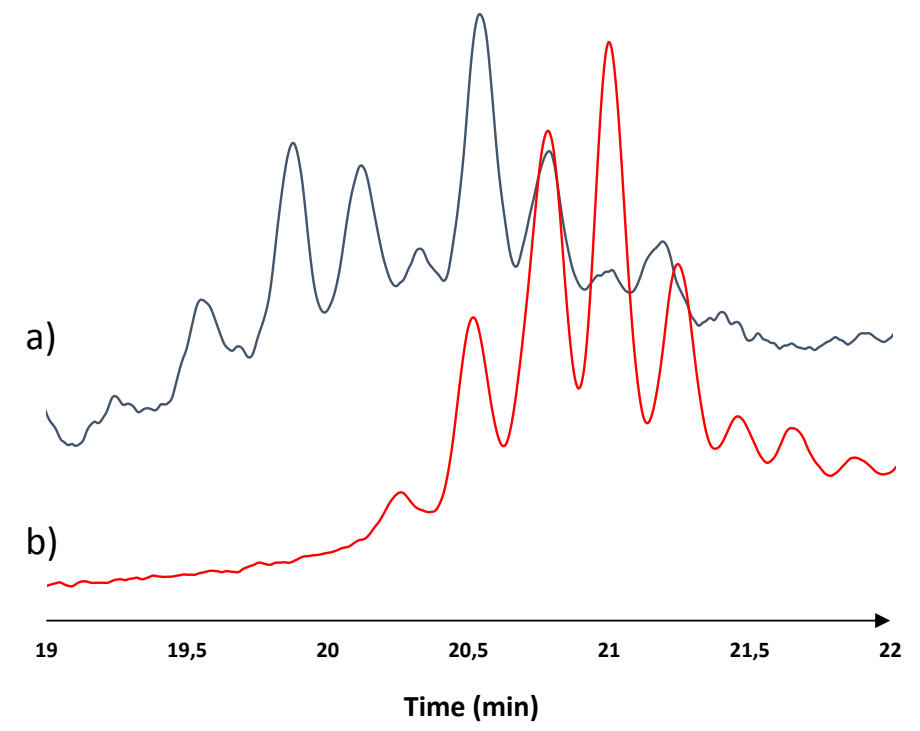

Figure S1: Overlaid chromatograms of the (a) u-hCG and (b) r-hCG for the first elution zone (19.0-21.8 min) obtained by HILIC-UV. Mobile phase: $0.1 \%$ TFA in $\mathrm{H}_{2} \mathrm{O} / \mathrm{ACN}$ mixture (v/v). Gradient: from 85 to $40 \%$ of $\mathrm{ACN}$ with a slope of $1.5 \% \mathrm{~min}^{-1}$. r- and $\mathrm{u}$-hCG: $1 \mathrm{mg} \mathrm{mL}^{-1}$ in water. Temperature: $60^{\circ} \mathrm{C}$. UV detection at $214 \mathrm{~nm}$.
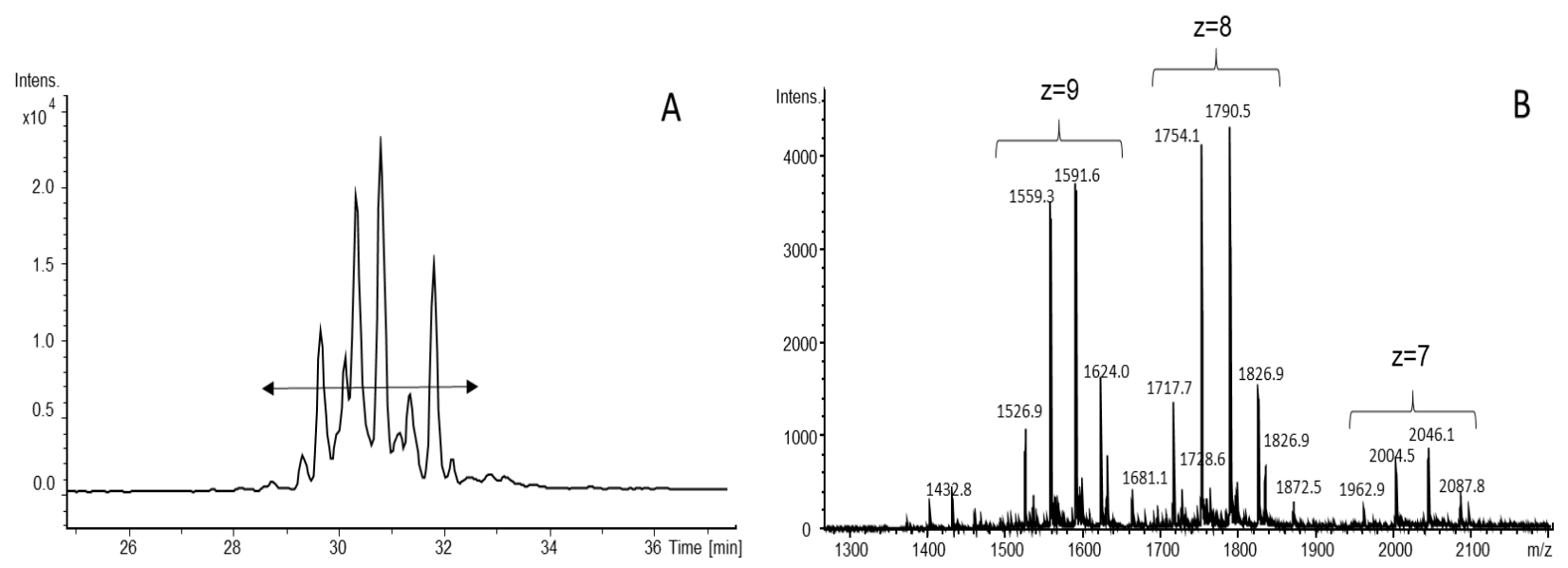

Figure S2: (A) BPC and (B) average MS spectrum between 28.5 and $32.5 \mathrm{~min}$ (see arrow) with the different charge states obtained by RPLC-HRMS for $r$-hCG. Mobile phase: $0.1 \%$ FA in $\mathrm{H}_{2} \mathrm{O} / \mathrm{ACN}$. Gradient: $4.5-31.5 \%$ of ACN with a slope of $0.5 \% \mathrm{~min}^{-1}$. Mass range: $1000-2500 \mathrm{~m} / \mathrm{z}$, full scan-positive mode. Temperature: $65^{\circ} \mathrm{C}$. r-hCG $\left(100 \mu \mathrm{g} \mathrm{mL}^{-1}\right)$ in water. Injected volume: $5 \mu \mathrm{L}$. Flow: $0.4 \mathrm{~mL} \mathrm{~min}^{-1}$. 
Table S1: MS parameters recommended by the manufacturer (Bruker Daltonics) for the analysis of macromolecules (with $\mathrm{m} / \mathrm{z}>1000$ ) and used for the analysis in RPLC-(qTOF) MS.

\begin{tabular}{lc} 
Transfer & \\
\hline isCID & $0.0 \mathrm{eV}$ \\
Funnel 1 RF & $400.0 \mathrm{Vpp}$ \\
Funnel 2 RF & $600.0 \mathrm{Vpp}$ \\
Hexapole RF & $800.0 \mathrm{Vpp}$ \\
\hline Quadrupole & \\
\hline lon energy & $5.0 \mathrm{eV}$ \\
Low mass & $300 \mathrm{~m} / \mathrm{z}$ \\
\hline Collision Cell & \\
\hline Collision Energy & $10.0 \mathrm{eV}$ \\
Collision RF & $1600 \mathrm{Vpp}$ \\
Transfer Time & $160.0 \mu \mathrm{s}$ \\
Pre-Pulse Storage & $15 \mu \mathrm{s}$
\end{tabular}

